\title{
Immunogenicity of the extracellular copper/zinc superoxide dismutase of the filarial parasite Acanthocheilonema viteae delivered by a two-phase vaccine strain of Salmonella typhimurium
}

\author{
CLAUS T.LATTEMANN ${ }^{1}$, ZHENG-XIN YAN ${ }^{1}$, ARNE MATZEN ${ }^{1,3}$, THOMAS F.MEYER TH $^{1,2}$ HEIKO APFEL $^{1,4}$ \\ ${ }^{1}$ Max-Planck-Institut für Biologie, Abteilung Infektionsbiologie, Spemannstrasse 34, D-72076 Tübingen, Germany \\ ${ }^{2}$ Max-Planck-Institut für Infektionsbiologie, Abteilung Molekulate Biologie, Monbijoustrasse 2, D-10117 Berlin, Germany
}

\section{SUMMARY}

The recombinant extracellular copper/zinc superoxide dismutase of the filarial parasite Acanthocheilonema viteae (AVSOD2) was cloned in an expression vector under control of the bacteriophage $T 7$ promoter and the resulting plasmid pLAT7 was introduced in tha aroA attenuated Salmonella typhimurium vaccine strain SL3261::pYZ84. This vaccine strain carries a chromosomally integrated two phase expression system containing inducible T7 RNA polymerase. The recombinant AVSOD2 was efficiently expressed, constituting up to $5 \%$ of the total bacterial protein. Furthermore, the plasmid vector containing the AVSOD2 cDNA was shown to be stable over a long period of time in the vaccine strain without antibiotic selection in vitro and in vivo. Jirds which were immunised orally with the recombinant vaccine strain expressing the A. viteae EC-SOD produced a strong humoral immune response.

Keywords filariasis, superoxide dismutase, vaccination, Salmonella, Acanthocheilonema viteae, Onchocerca volvulus

Correspondence: $\mathrm{H}$. Apfel

Received: 18 May 1998

Accepted for publication: 11 September 1998

${ }^{3}$ Present address: IBT1, Forschungszentrum Jülich, D-52428 Jülich, Germany

${ }^{4}$ Present address: Creatogen Live Vaccines GmbH, Ulmer Strasse 160A, D-86156 Augsburg, Germany

\section{INTRODUCTION}

In the course of infection, many pathogens are confronted by activated oxygen metabolites generated by activated leukocytes which undergo an 'oxidative burst'. Consequently the expression of high levels of antioxidant enzymes is implicated to play an important role in immune evasion of pathogenic bacteria and parasites (Bannister et al. 1987, Callahan et al. 1988, Maizels et al. 1993). Superoxide Dismutases (SODs) protect cells by inactivating the superoxide radical $\left(\cdot \mathrm{O}_{2}{ }^{-}\right)$which is able to oxidize biological membranes and proteins (Brunori \& Rotilio 1984). According to their metal cofactors, SODs are grouped into three classes, Fe SODs, Mn SODs, and CuZn SODs, the latter of which are primarily found in eukaryotes. Two SOD subtypes have been characterised, cytoplasmic (CY-SOD) and extracellular (EC-SOD) SOD. In various organisms SOD expression is correlated with extrinsic or intrinsic oxidative stress, and expression of SOD is up-regulated in response to the oxygen tension in prokaryotes and eukaryotes (Bannister et al. 1987). The immunoprotective potential of a vaccination with SOD has been evaluated for several bacterial species, leading to a protective immune response in experimental infection with Mycobacterium leprae (Gelber et al. 1994) and Listeria monocytogenes (Hess et al. 1997). SODs recently have also been characterized for several filarial species (Henkle-Dührsen et al. 1991, James et al. 1994, Tang et al. 1994) and there is evidence that filarial parasites release SOD activity into the surrounding environment (Batra et al. 1990, Henkle-Dührsen et al. 1991, Tang et al. 1994). Thus, SODs may participate in immune evasion of filarial infections and therefore are considered as a potential target molecule for an anti-filarial vaccine.

Attenuated vaccine strains of Salmonella typhimurium 
and $S$. typhi have been shown to be efficient carriers for the delivery of recombinant antigens. Salmonella vaccine strains expressing various recombinant antigens have been shown to elicit humoral and $\mathrm{T}$ cell-dependent immune responses against these antigens in animals and humans (Brown et al. 1987, Turner et al. 1993, Khan et al. 1994, Maskell et al. 1987, Hopkins et al. 1995). The immune response induced by such recombinant Salmonella vaccines was shown to be protective against viral, bacterial, and protozoan infections in several animal models (Poirier et al. 1988, Tite et al. 1990, Yang et al. 1990, Oyston et al. 1995, Gómez-Duarte et al. 1997, Hess et al. 1997). To evoke an immune response, the recombinant antigen has to be presented in large amounts within a certain period of time. Nevertheless, the constitutive high level expression of foreign antigen often interferes with the viability of the recombinant Salmonella strain, thus resulting in the reduced stimulation of the immune system. Previously, we have reported the design of an attenuated $S$. typhimurium aroA vaccine strain by a rational approach combining the bacteriophage $\mathrm{T} 7$ polymerase/promoter system (Tabor \& Richardson 1985) and a temperature-dependent phase variation system (Yan \& Meyer 1994, 1996). This system guarantees the stable expression of foreign antigens in vivo since the vaccine strain population exists as two subpopulations. The non-expressing population is actively dividing and propagating like the parental vaccine strain, while the phase variable switching of a small proportion of this population constantly renews the subpopulation which expresses the recombinant antigen.

In this communication, we investigate the delivery of the recombinant EC-SOD of the small mammal filaria Acanthocheilonema viteae (AVSOD2, Genbank accession number AJ010164) expressed in the aroA attenuated S. typhimurium vaccine strain SL3261::pYZ84 (Yan \& Meyer 1996). The natural parasite host system A. viteaeMeriones unguiculatus, is an important animal model to study the interaction of filarial parasites with the final host (Lok \& Abraham 1992). We have analysed the immune response in jirds induced by oral inoculation of SL3261::pYZ84 expressing the recombinant AVSOD2, the stability of antigen expression in vitro and in vivo was determined, and the humoral immune response of the jirds was analysed.

Prior to vaccination, the expression level of recombinant AVSOD2 was examined in the vaccine strain SL3261::pYZ84 transformed with the expression vector pLAT7 which carries the recombinant AVSOD2. SDSPAGE analysis demonstrates the efficient expression of
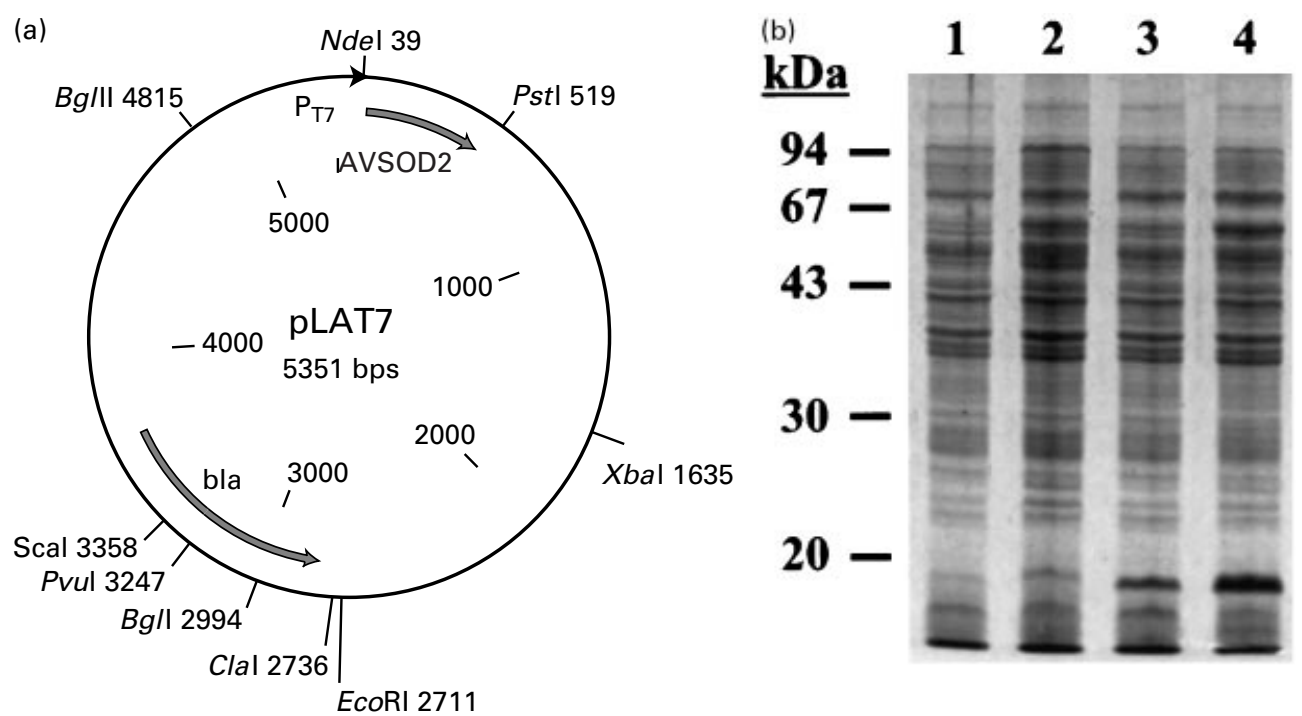

Figure 1 (a) Construction of the expression vector for the AVSOD2. The cDNA of the A. viteae AVSOD2 (Lattemann, Matzen \& Apfel, in preparation) was subcloned into the expression vector pYZ97 (Yan \& Meyer 1994) under control of the bacteriophage T7 promoter ( $\left.\mathrm{P}_{\mathrm{T} 7}\right)$. A fragment of the cDNA was amplified by PCR with the primers MAT2 $\left(5^{\prime}\right.$-GCGCCCCCGGGCATATGGCACGTGAAAGCAATTCTAAA-3 $)$ and HA17 (5'-GGGCTGCAGACCAGCATTACCGGTTTTCA-3'). The sense primer MAT2 was designed to omit the putative signal peptide and carries a NdeI restriction site so that the cDNA could be directly fused into the start codon of pYZ97. Due to the PCR strategy which was initially chosen to amplify the AVSOD2 cDNA from total A. viteae cDNA, the cDNA was truncated by 42 nucleotides at the $3^{\prime}$-end of the coding region. (b): Expression of recombinant AVSOD2 in S. typhimurium SL3261 Salmonella strains SL3261::pYZ84 and SL3261::pYZ84/pLAT7 expressing recombinant AVSOD2 were cultivated overnight at $28^{\circ} \mathrm{C}$ and $37^{\circ} \mathrm{C}$. Expression of recombinant AVSOD2 was determined by Coomassie stained SDS-PAGE loading equal amounts of bacteria in each lane. Lane 1: SL3261::pYZ84 (28 $\left.{ }^{\circ} \mathrm{C}\right)$; Lane 2: SL3261::pYZ84 (37 $\left.{ }^{\circ} \mathrm{C}\right)$; Lane 3: SL3261::pYZ84/pLAT7 (28 ${ }^{\circ}$ ); Lane 4: SL3261::pYZ84/pLAT7 $\left(37^{\circ} \mathrm{C}\right)$. 
the recombinant AVSOD2 migrating to $17 \mathrm{kDa}$ (Figure 1). Cultivation of the bacteria at $37^{\circ} \mathrm{C}$ resulted in clearly enhanced expression of recombinant AVSOD2 leading to the accumulation of at least $5 \%$ of the cellular protein. Background expression of SOD is observed upon cultivation at $28^{\circ} \mathrm{C}$. Next, we assessed in vitro the stability of the expression vector pLAT7 by passaging the vaccine strain four times for $12 \mathrm{~h}$ in medium without antibiotic selection, and found that $85 \%$ of the bacteria retained the construct (data not shown). To determine the in vivo stability of
pLAT7, liver and spleen of jirds orally immunized with SL3261::pYZ84/pLAT7 were homogenized and the number of Salmonella recovered was determined (Figure 2). The total number of bacteria varied substantially among the individual animals, with a mean of $590 \mathrm{cfu}$ in liver at day 7 which had declined to $20 \mathrm{cfu}$ day 14 . The spleen was colonized with an average of $14 \mathrm{cfu}$ at day 7. At day 21 no bacteria could be recovered from liver or spleen, confirming the attenuation of this strain which allows safe antigen delivery. The proportion of bacteria resistant to

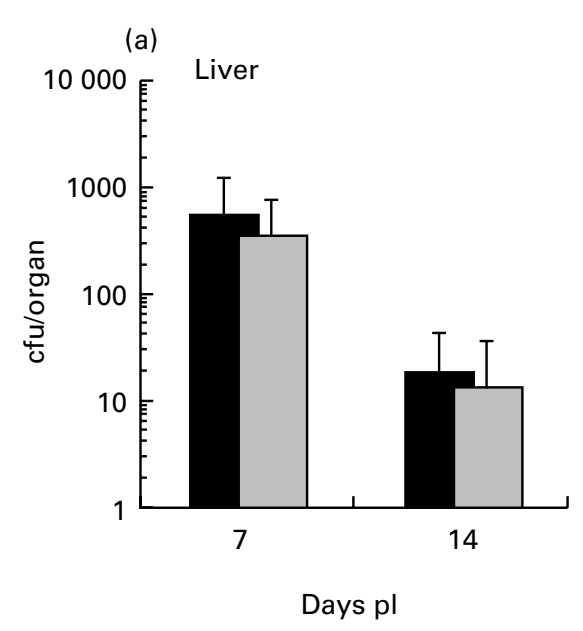

(c)

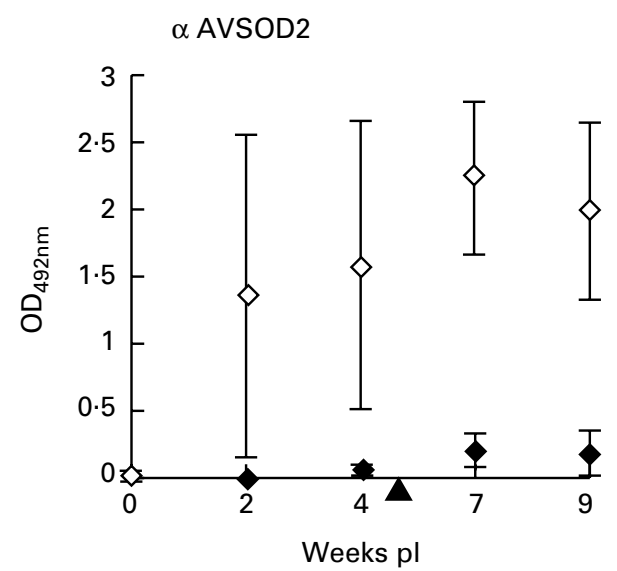

(b)

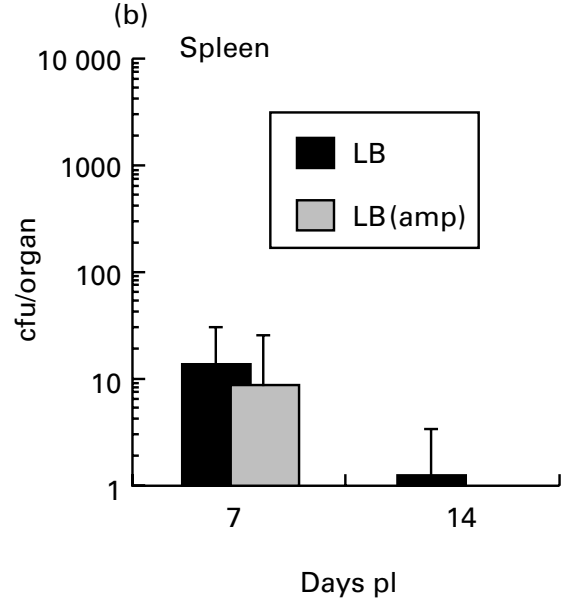

(d)

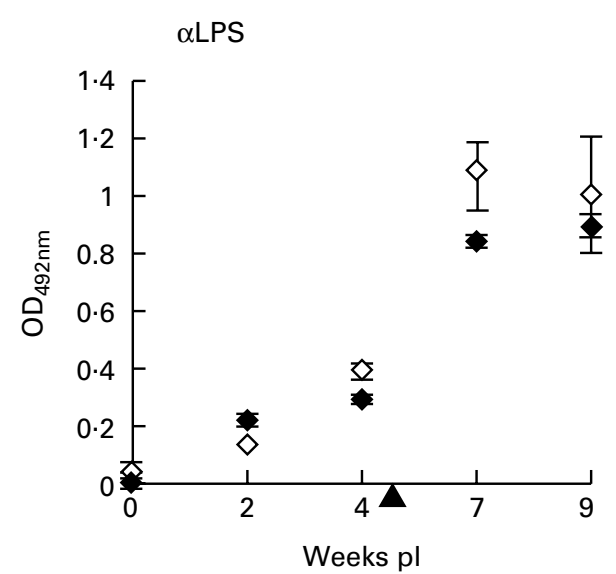

Figure 2 (a,b): Recovery of SL3261: pYZ84/pLAT7 from liver and spleen of immunized jirds. The organs of jirds orally immunized with $5 \times 10^{8}$ cfu SL3261::pYZS4/pLAT7 were removed at day $7(n=6$, two independent experiments) and at day $14(n=3)$, homogenized and plated on LB plates with and without ampicillin. Viable cell counts were performed after cultivation overnight at $28^{\circ} \mathrm{C}$. (c,d): Vaccination of jirds with $\mathrm{S}$. Typhimurium expressing recombinant AVSOD2. The humoral immune response of jirds orally vaccinated with $5 \times 10^{8}$ cfu SL3261::pYZ84/pLAT7 (open diamonds, $n=6$ ), SL3261::pYZ84 (filled diamonds, $n=5$ ) was determined by ELISA against purified recombinant AVSOD2 (c) and Salmonella LPS (d) A booster immunization was performed on all jirds at week 5. Blood samples of the immunized jirds were collected at week 2, 4, 7 and 9 after the first immumization from the retroorbital venous plexus. These are representative results. One non-responding animal was excluded from the study. 
ampicillin was about $70 \%$ of the total recovered at each time point of isolation. Additionally, $96 \%$ of the bacteria isolated from LB plates were resistant to ampicillin. These results demonstrate that pLAT7 is stable under in vitro and in vivo conditions and therefore is suitable for vaccination studies. The humoral immune response of jirds immunised orally with SL3261::pYZ84/pLAT7 was assessed by ELISA using a rabbit antiserum raised against jird total immunoglobulin and a goat anti rabbit peroxidase conjugate (Sigma) to detect the bound antibodies (Figure 2). After the first immunization, the immunized jirds developed a strong humoral immune response against recombinant AVSOD2, and one week after the booster immunisation this antibody response reached a maximum level before decreasing slightly at week nine. Control animals which were treated with SL3261::pYZ84 or saline produced no antibodies against AVSOD2 (Figure 2c). The antibody response against Salmonella LPS was similar in the groups of animals which were immunized either with SL3261::pYZ84/pLAT7 or SL3261::pYZ84, while no reaction was observed in animals which received saline (Figure 2d). Additionally, the sera of immunized jirds were analysed by Western blot against total worm homogenate of A. viteae and Onchocerca volvulus. These sera specifically recognised both the CYSOD and the EC-SOD in both worm homogenates (data not shown).

Our results demonstrate that oral immunization with attenuated $S$. typhimurium strains expressing recombinant filarial antigens is a promising strategy for the development of anti-filarial vaccines. We have efficiently expressed the AVSOD2 in the aroA $S$. typhimurium vaccine strain SL3261::pYZ84 to generate a strain which stimulates a strong humoral immune response against the enzyme in vaccinated jirds. Only three out of 16 animals produced negligible levels of antibodies against the AVSOD2 whereas the other animals responded well to the recombinant antigen in ELISA. The immune sera were seen to specifically recognise AVSOD2 by Western blot analysis of total worm homogenate, thus confirming the results obtained in ELISA. Our results contrast the finding from natural infections of jirds with A. viteae, where only a small proportion of animals produce antibodies against recombinant AVSOD2 (Lattemann, Matzen \& Apfel in preparation). Immunization studies with purified recombinant EC-SOD from $O$. volvulus that was applied with different adjuvants also failed to induce SOD-specific immune response in jirds (Lucius 1995). The induction of antibody response by recombinant Salmonella has been shown to depend on the amount of antigen produced by recombinant vaccine strains for the efficient stimulation of an immune response (Fayolle et al. 1994). However, overexpression of foreign antigens is often toxic for the antigen producing cell (Dong, Nilsson \&
Kurland 1995), and bacteria constitutively producing high levels of foreign antigen are often rapidly cleared in vivo by the immune system or lose the expression plasmid (Khan et al. 1994, Oyston et al. 1995, author's unpublished observations). To avoid this, we successfully utilized the natural phenomenon of phase variation to generate a heterogeneous population within the vaccine strain which guarantees the continuous formation of an antigen-expressing pool of bacteria (Yan \& Meyer 1994, 1996). Due to this strategy, the Salmonella expressed recombinant AVSOD2 at high rates and did not lose the plasmid. Most importantly, overexpression of the recombinant AVSOD2 did not impair the invasion and colonization properties of SL3261::pYZ84/ pLAT7 compared to the wild type strain, as illustrated by the similar immune response against Salmonella LPS in animals immunized with both strains.

The effector mechanisms of protective anti-filarial immunity are not yet understood, however, some studies underline the importance of the mode of antigen presentation for the design of anti-filarial vaccines. In experimental infection with Brugia malayi, mice of the $\mathrm{H}-2 \mathrm{~d}$ haplotype do not produce antibodies against gp29, the cuticular glutathione peroxidase (Cookson et al. 1992). Also immunizations with parasite extract administered in incomplete Freund's adjuvant failed to induce gp29 specific immune response in this mice, while delivery of recombinant gp29 by live attenuated Salmonella could at least partially overcome this restriction in H-2d mice (Chacón et al. 1996). Chacón et al. contribute this effect to the different mode of antigen presentation. Despite the fact that Salmonella remain inside phagolysosomal compartments, infected macrophages have been shown to present antigenic peptides by both MHC class I and class II molecules (Pfeifer et al. 1993, Turner et al. 1993, Wick et al. 1994). Consequently, both cytotoxic and humoral immune responses are potently triggered by recombinant Salmonella vaccines. Furthermore, Salmonella are capable of inducing a broad spectrum of antibody isotypes against helminth antigens, indicating that $\mathrm{T}$ helper cells expressing Th1 and Th2 type cytokines are induced (Chacón et al. 1996, Comoy et al. 1997). To investigate the potential of recombinant AVSOD2 delivered by SL3261::pYZ84 to protect jirds from a challenge infection with $A$. viteae, we have performed protection studies in jirds. Preliminary results show a reduction of the worm burden of about $30 \%$ in jirds vaccinated with SL3261::pYZ84/pLAT7 in comparison to the control groups which received the non recombinant vaccine strain or saline (unpublished results). This indicates that the EC-SOD indeed is a promising vaccine candidate for an anti-filarial vaccine. Protective immunity may be further enhanced by the application of pools of Salmonella expressing a repertoire of antioxidant enzymes of filariae, and our future studies will 
thus be aimed at optimizing antigen delivery of Salmonella by pooling filarial antigens. We also plan to express the AVSOD2 in different bacterial compartments in order to assess whether periplasmic or surface expression has beneficial effects on antigen presentation and the immune response.

\section{ACKNOWLEDGEMENTS}

The authors wish to thank Dr S. Gray-Owen for reviewing this manuscript.

\section{REFERENCES}

Bannister J.V., Bannister W.H. \& Rotilio G. (1987) Aspects of the structure, function, and applications of superoxide dismutase. Critical Reviews in Biochemistry 22, 111-180

Batra S., Chatterjee R.K. \& Srivastava V.M. (1990) Antioxidant enzymes in Acanthocheilonema viteae and effect of antifilarial agents. Biochemical Pharmacology 40, 2363-2369

Brunori M. \& Rotilio G. (1984) Biochemistry of oxygen radical species. Methods in Enzymology 105, 22-35

Brown A., Hormaeche C.E., de Hormaeche R.D. et al. (1987) An attenuated aroA Salmonella typhimurium vaccine strain elicits humoral and cellular immunity to cloned $\beta$-galactosidase in mice. Journal of Infectious Diseases 155, 86-92

Callahan H.L., Crouch R.K. \& James E.R. (1988) Helminth antioxidant enzymes: a protective mechanism against host oxidants? Parasitology Today 8, 218-225

Chacón M.R., Londoño P., Dougan G. et al. (1996) Heterologous expression of the cuticular glutathione peroxidase of lymphatic filariae in an attenuated vaccine strain of Salmonella typhimurium abrogates H-2 restriction of specific antibody responses. Parasite Immunology 18, 307-316

Comoy E.E., Capron A. \& Thyphronitis G. (1997) In vivo induction of type 1 and type 2 immune responses against protein antigens. International Immunology 9, 523-531

Cookson E., Blaxter M.L. \& Selkirk M.E. (1992) Identification of the major soluble cuticular glycoprotein of lymphatic filarial parasites (gp29) as a secretory homologue of glutathione peroxidase. Proceedings of the National Academy of Sciences, USA 89, 5837-5841

Dong H., Nilsson L. \& Kurland C.G. (1995) Gratuitous overexpression of genes in Escherichia coli leads to growth inhibition and ribosome destruction. Journal of Bacteriology 177, 1497-1504

Fayolle C., O'Callaghan D., Martineau P. et al. (1994) Genetic control of antibody responses induced against an antigen delivered by recombinant attenuated Salmonella typhimurium. Infection and Immunity 62, 4310-4319

Gelber R.H., Mehra V., Bloom B. et al. (1994) Vaccination with pure Mycobacterium leprae proteins inhibits $M$. leprae multiplication in mouse footpads. Infection and Immunity 62, 4250-4255

Gómez-Duarte O.G., Lucas B., Yan Z.X. et al. (1998) Protection of mice against gastric colonization by Helicobacter pylori by single oral dose with attenuated Salmonella typhimurium producing urease subunits A and B. Vaccine. 16, 460-471

Henkle-Dührsen K., Liebau E., Müller S. et al. (1991) Characterization and molecular cloning of a copper-zinc superoxide dismutase from the human pathogen Onchocerca volvulus. Infection and Immunity 59, 2063-2069
Hess J., Dietrich G., Gentschev I. et al. (1997) Protection against murine listeriosis by an attenuated recombinant Salmonella typhimurium vaccine strain that secretes the naturally somatic antigen superoxide dismutase. Infection and Immunity 65, 1286-1292

Hopkins S., Kraehenbuhl J.-P., Schödel F. et al. (1995) A recombinant Salmonella typhimurium vaccine induces local immunity by four routes of immunization. Infection and Immunity 63, 3279-3286

Khan C.M., Villareal-Ramos B., Pierce R.J. et al. (1994) Construction, expression and immunogenicity of the Schistosoma mansoni P28 glutathione S-transferase expressed as a genetic fusion to tetanus toxin fragment $\mathrm{C}$ in a live attenuated vaccine strain of Salmonella. Proceedings of the National Academy of Sciences, USA 91, 1126111265

Lattemann C.T., Matzen A. \& Apfel H. Up-regulation of extracellular copper/zinc superoxide dismutase mRNA after transmission of the filarial parasite Acanthocheilonema viteae in the vertebrate host Meriones unguiculatus (manuscript in preparation)

Lok J.B. \& Abraham D. (1992) Animal models for the study of immunity in human filariasis. Parasitology Today 8, 168-171

Lucius R. (1995) Screening of recombinant filarial antigens in animal models: How can we shape protective antigens? The Greene Sheets. Edna McConnell Clark Foundation, USA volume 3

Maskell D., Sweeney K.J., O'Callaghan D. et al. (1987) Salmonella typhimurium aroA mutants as carriers of the Escherichia coli heat labile enterotoxin B subunit to the murine secretory and systemic immune systems. Microbial Pathogenesis 2, 211-221

Maizels, R.M., Bundy, D.A.P., Selkirk M.E. et al. (1993) Immunological modulation and evasion by helminth parasites in human populations. Nature 365, 797-895

Oyston P.C.F., Williamson E.D., Leary S.E.C. et al. (1995) Immunization with live recombinant Salmonella typhimurium aroA producing F1 antigen protects against plague. Infection and Immunity 63, 563-568

Pfeifer J.D., Wick M.J., Roberts R.L. et al. (1993) Phagocytic processing of bacterial antigens for class I MHC presentation to T cells. Nature 261, 359-362

Poirier T.P., Kehoe M.A. \& Beachey E.H. (1988) Protective immunity evoked by oral administration of attenuated Salmonella typhimurium expressing cloned streptococcal M protein. Journal of Experimental Medicine 168, 25-32

Tabor S. \& Richardson C. (1985) A bacteriophage T7 RNA polymerase/promoter system for controlled exclusive expression of specific genes. Proceedings of the National Academy of Sciences, USA 82, 1074-1078

Tang L., Ou X., Henkle-Dührsen K. et al. (1994) Extracellular and cytoplasmic $\mathrm{CuZn}$ superoxide dismutase from Brugia lymphatic filarial nematode parasites. Infection and Immunity 62, 961-967

Tite J.P., Gao X.-M., Hughes-Jenkins C.M. et al. (1990) Anti-viral immunity induced by recombinant nucleoprotein of influenza A virus: III. Delivery of recombinant nucleoprotein to the immune system using attenuated Salmonella typhimurium as live carrier. Immunology 70, 540-546

Turner S.J., Carbone F.R. \& Strugnell R.A. (1993) Salmonella typhimurium $\triangle$ aroA $\triangle a r o D$ mutants expressing a foreign recombinant protein induce specific major histocompatibility complex class-I restricted T-lymphocytes in mice. Infection and Immunity 61, 5374-5380

Wick M.J., Harding C.V., Normark S.J. et al. (1994) Parameters that influence the efficiency of processing antigenic epitopes expressed in Salmonella typhimurium. Infection and Immunity 62, 4542-4548.

Yan Z.X. \& Meyer T.F. (1994) The variation of antigen expression: a natural phenomenon utilized for construction of live recombinant Salmonella vaccines. Behring Institute Mitteilungen 95, 49-56 
Yan Z.X. \& Meyer T.F. (1996) Mixed population approach for vaccination with live recombinant Salmonella strains. Journal of Biotechnology 44, 197-201

Yang D.M., Fairweather N., Button L.L. et al. (1990) Oral Salmonella typhimurium (Aro-) vaccine expressing a major leishmanial surface protein (gp63) preferentially induces $\mathrm{T}$ helper cells and protective immunity against Leishmaniasis. Journal of Immunology 145, 22812285 